\title{
A study of Earth-Moon Trajectories based on Analytical Expressions for the Velocities Increments
}

\author{
Luiz Arthur Gagg Filho \\ Departamento de Matemática, Instituto Tecnológico de Aeronáutica - ITA, São José dos \\ Campos, SP.
}

\section{Sandro da Silva Fernandes ${ }^{2}$}

Departamento de Matemática, Instituto Tecnológico de Aeronáutica - ITA, São José dos Campos, SP.

\begin{abstract}
A study of Earth-Moon bi-impulsive trajectories is presented in this paper. The dynamic model utilized to describe the motion of the space vehicle is the classic planar circular restricted three-body problem (PCR3BP). The velocities increments are obtained through analytical expressions, which are derived from the development of the complete Jacobi Integral expression. In order to determine the trajectories, a two-point boundary value problem (TPBVP) with prescribed Jacobi Integral is formulated. In this way, internal and external trajectories are determined with several times of flight. The results show that this method facilitates the determination of Earth-Moon trajectories with large time of flight; it offers a better intuition of the problem since the Jacobi Integral is specified; it allows several kind of studies since all Earth-Moon trajectories can be obtained; and, it provides a good estimate for optimization algorithms which can be, eventually, applied in order to minimize the total fuel consumption.
\end{abstract}

Keywords. Earth-Moon trajectories, Jacobi Integral, three-body problem, multiple revolution trajectories, bi-impulsive trajectories.

\section{Introduction}

The problem of transferring a space vehicle from one orbit to another has been growing in importance in last decades. The Grail mission is one of the latest mission to the Moon and its objective is to study the lunar gravitational field [7] in order to find some information about the origin of the Moon. Besides the science, the transferring of a space vehicle is used in commercial applications such as the maintenance of satellites. In the majority of the applications, the actuators of the space vehicle are assumed to be

1 luizarthur.gagg@gmail.com

2 sandro@ita.br 
impulsive, which means that they produce an instantaneous velocity increment to put the vehicle in the desired trajectory. The determination of impulsive trajectories in complex dynamic models has become more studied since the second half of the 20th century until nowadays. In this way, two kinds of studies can be highlighted. The first one is characterized by using optimization algorithms, especially those that use gradient methods in order to minimize the fuel consumption, which is represented by the total velocity increment [5]. The second kind of study consists in exploring more deeply the dynamic of the restricted three body problem, and, uses the notion of weak stability boundary [1] or the invariant manifolds [4]. Da Silva Fernandes and Marinho [3] have used a gradient method with the Newton-Raphson algorithm to find optimal Earth-Moon trajectories considering the gravitational influence of the Sun. However, these optimizations processes have been proved to be sensitive to the initial conditions. On the other hand, if external trajectories or internal trajectories with a large time of flight are desirable, the known patched-conic approximation could not be used as initial condition. Defining the initial conditions in these cases requires a deep intuition of the problem, which is, sometimes, impossible to acquire due to the several number of parameters to adjust, and, to the natural sensitivity of the orbital dynamic problems. Therefore, a multidimensional search is usually needed to determine the initial conditions. The greater the number of parameters to adjust larger is the dimension of the search space. Topputo [6], for instance, has done a search in a four dimensional space to generate the initial conditions in his algorithm to obtain optimal Earth-Moon trajectories with different times of flight. A reduction in the dimension of the search space decreases significantly the processing time. The work of Da Silva Fernandes and Marinho [2], likewise Topputo [6], studies Earth-Moon trajectories with different times of flight and, also, has four variables to adjust as initial condition.

The present work is based on the paper of Da Silva Fernandes and Marinho [2], but the initial conditions of both velocity increments are obtained through analytical expressions originated by developing the Jacobi Integral in the planar circular restricted three body problem (PCR3BP). A new two-point boundary value problem (TPBVP) is formulated, where the search space of the initial conditions is reduced.

\section{Objective}

The main purpose of this work is to determine Earth-Moon trajectories with large times of flight in the classic planar circular restricted three-body problem (PCR3BP) through a two-point boundary value problem with a prescribed value of the Jacobi Integral.

\section{Methods}

The purpose of this formulation is motivated by the orbital transfer problem, in which is desirable to transfer a space vehicle with infinitesimal mass from a low Earth orbit (LEO) to a low Moon orbit (LMO) by the application of two velocities increments. These increments are assumed to be impulsive and tangential to the terminal orbits. The 
first velocity increment is applied in the LEO and it accelerates the space vehicle putting it in a transfer trajectory. The second velocity increment is applied in the arrival near the Moon and it slowdowns the space vehicle, circularizing its movement in the LMO. The terminal orbits are assumed to be circular. In this work, the dynamical model utilized to describe the motion of the vehicle is the classic PCR3BP. With this problem in mind, the following mathematical formulation is the foundation to construct a TPBVP in order to solve it. In this way, the next topics show the dynamic model of the PCR3BP and the development of the Jacobi Integral in order to obtain analytical expressions for the velocities increments. Finally, the TPBVP is stated.

Assume that the space vehicle has coordinates $\left(x_{P}, y_{P}\right)$ in an inertial reference frame with origin at the CM point (center of mass of the Earth-Moon system), where the subscript $P$ defines quantities related to the space vehicle. The motion of the space vehicle is then described by the following differential equations:

$$
\begin{aligned}
& \ddot{x}_{p}=-\frac{\mu_{E}}{r_{E P}^{3}}\left(x_{p}-x_{E}\right)-\frac{\mu_{M}}{r_{M P}^{3}}\left(x_{p}-x_{M}\right) \\
& \ddot{y}_{p}=-\frac{\mu_{E}}{r_{E P}^{3}}\left(y_{p}-y_{E}\right)-\frac{\mu_{M}}{r_{M P}^{3}}\left(y_{p}-y_{M}\right),
\end{aligned}
$$

where the subscripts $E$ and $M$ define quantities related, respectively, to the Earth and to the Moon. Thus, $\mu_{E}$ is the gravitational parameter of the Earth, and, $\mu_{M}$ is the gravitational parameter of the Moon. The operator - above the variables represents time derivatives quantities. $r_{E P}$ and $r_{M P}$ are the radial distances of the vehicle to the Earth and to the Moon respectively.

\subsection{Analytical expressions for the velocities increments}

Consider the complete expression of the Jacobi Integral as below:

$$
J\left(\xi_{p}, \eta_{p}, \dot{\xi}_{p}, \dot{\eta}_{p}\right)=2\left(\frac{1}{2} \omega^{2}\left(\xi_{p}^{2}+\eta_{p}^{2}\right)+\frac{\mu_{E}}{r_{E P}}+\frac{\mu_{M}}{r_{M P}}\right)-\left(\dot{\xi}_{p}^{2}+\dot{\eta}_{p}^{2}\right),
$$

where $J\left(\xi_{p}, \eta_{p}, \dot{\xi}_{p}, \dot{\eta}_{p}\right)$ is the Jacobi Integral, $\left(\xi_{p}, \eta_{p}\right)$ and $\left(\dot{\xi}_{p}, \dot{\eta}_{p}\right)$ are the position and velocity coordinates of the space vehicle in a rotating reference frame $G \xi \eta$, which contains the orbital plane of the Moon and with origin at the CM point. The $\xi$ axis points toward the Moon at each time instant $t$, and, the $\eta$ axis is perpendicular to the $\xi$ axis.

From the Jacobi Integral, one finds that the first velocity increment $\Delta v_{1}$, applied in the LEO, is given by 


$$
\begin{aligned}
& \Delta v_{1}=\left(\omega r_{E P}(0)\right)-\sqrt{\frac{\mu_{E}}{r_{E P}(0)}} \pm \\
& \sqrt{2\left[\frac{1}{2} \omega^{2}\left(\left(r_{E P}(0)\right)^{2}+\left(\frac{\mu D}{1+\mu}\right)^{2}-2\left(\frac{\mu D}{1+\mu}\right)\left(r_{E P}(0) \cos \left(\theta_{E P}(0)\right)\right)+\frac{\mu_{E}}{r_{E P}(0)}+\frac{\mu_{M}}{r_{M P}(0)}\right]-J\left(\xi_{p}, \eta_{p}, \dot{\xi}_{p}, \dot{\eta}_{p}\right)\right.}
\end{aligned}
$$

where $D$ is the mean distance from the Earth to the Moon, $\omega=\sqrt{\left(\mu_{M}+\mu_{E}\right) / D^{3}}$ is the angular velocity of the Moon around $\mathrm{CM}$, and $\theta_{E P}$ is the angle between the position vector of the space vehicle with respect to the Earth and the $x$ axis of the inertial reference frame.

By an analog development from the Jacobi Integral, one finds that the second velocity increment $\Delta v_{2}$, applied in the LMO, is given by:

$$
\begin{gathered}
\Delta v_{2}=-\sqrt{\frac{\mu_{M}}{r_{M P}(T)}} \mp \\
\left\{\left(\omega r_{M P}(T)\right) \pm \sqrt{\left[\omega^{2}\left(\left(r_{M P}(T)\right)^{2}+\left(\frac{D}{1+\mu}\right)^{2}+2\left(r_{M P}(T)\right)\left(\frac{D}{1+\mu}\right)\left(\cos \left(\theta_{M P}(T)-\omega T\right)\right)\right)+\frac{2 \mu_{E}}{r_{E P}(T)}+\frac{2 \mu_{M}}{r_{M P}(T)}\right]-J\left(\xi_{p}, \eta_{p}, \dot{\xi}_{p}, \dot{\eta}_{p}\right)}\right\}
\end{gathered},
$$

where $\theta_{M P}$ is the angle between the position vector of the space vehicle with respect to the Moon and the $x$ axis of the inertial reference frame.

The analytical expressions given by Eqs. (4) and (5) provide a way to estimate the velocities increments $\Delta v_{1}$ and $\Delta v_{2}$, respectively, assuming that a value of the Jacobi Integral is prescribed. An analysis of magnitude order shows that the terms which depend on $\theta_{E P}(0)$ and $\theta_{M P}(T)$ can be neglected, since their contributions to the velocities increments are insignificant. Hence, the following approximations are suggested in order to determine the velocities increments: $\cos \left(\theta_{E P}\right)=1$ in Eq. (4); and, $\cos \left(\theta_{M P}(T)-\omega T\right)=1$ in Eq. (5).

\subsection{The two point boundary value problem (TPBVP)}

Based on the analytical expressions for the velocities increments determined in the preceding section, a TPBVP is formulated for the PCR3BP in order to obtain Earth-Moon trajectories with a prescribed value of the Jacobi Integral. The TPBVP is enunciated as below:

"For a value of the Jacobi Integral $C=J\left(\xi_{p}, \eta_{p}, \dot{\xi}_{p}, \dot{\eta}_{p}\right)$, determine the set of variables $\left(\theta_{E P}(0), \theta_{M P}(T), T\right)$ that satisfies the specified final constraints of the differential equations (1) and (2)."

Note that this problem has three variables to be iterated. However, the search space of the variable $\mathrm{T}$ is reduced when the total time of flight of the space vehicle is relatively known. Moreover, this formulation allows setting the Jacobi Integral previously which gives three advantages: increases the problem intuition since the Jacobi Integral already provides, initially, an idea of the total fuel consumption through Eqs. (4) and (5); the determination of 
the desired trajectories is facilitated, especially for those trajectories with large time of flight; and, a detailed study of the Kepler's energy of the space vehicle in the arrival of the low Moon orbit (LMO) can be developed.

\section{Results}

Table 1 shows the data used in the numerical simulations. The LEO altitude is $167 \mathrm{~km}$, and, the LMO altitude is $100 \mathrm{~km}$. The space vehicle can arrive in the LMO in the clockwise or counterclockwise sense. In this paper, only clockwise arrivals are considered. Several trajectories are obtained considering the Jacobi Integral in the interval $[1,2.93] \mathrm{km}^{2} / \mathrm{s}^{2}$. Table 2 shows some results of different trajectories for the same Jacobi Integral value $(C=$ $1.20 \mathrm{~km}^{2} / \mathrm{s}^{2}$ ). The trajectories are obtained through the TPBVP described in the previous section. Figures 1-4 illustrate others trajectories with different configurations of time of flight and/or Jacobi Integral values.

Table 1: Parameters specification

\begin{tabular}{|l|c|}
\hline$\omega_{M}$ (angular velocity of the Moon with respect to the Earth) & $2.649 \times 10^{-6} \mathrm{rad} / \mathrm{s}$ \\
\hline$v_{M}$ (linear velocity of the Moon with respect to the Earth) & $1.018 \mathrm{~km} / \mathrm{s}$ \\
\hline$R_{E}$ (Earth's radius) & $6378.2 \mathrm{~km}$ \\
\hline$R_{M}$ (Moon's radius) & $1738 \mathrm{~km}$ \\
\hline$\mu_{E}$ & $398600 \mathrm{~km}^{3} / \mathrm{s}^{2}$ \\
\hline$\mu_{M}$ & $4902.83 \mathrm{~km} / \mathrm{s}^{2}$ \\
\hline $\mathrm{D}$ & $384400 \mathrm{~km}$ \\
\hline
\end{tabular}

Table 2: Trajectories with different times of flight at $C=1.20 \mathrm{~km}^{2} / \mathrm{s}^{2}$.

\begin{tabular}{|c|c|c|c|c|c|}
\hline$\Delta v_{1}[\mathrm{~km} / \mathrm{s}]$ & $\Delta v_{2}[\mathrm{~km} / \mathrm{s}]$ & $\Delta v_{\text {TOTAL }}[\mathrm{km} / \mathrm{s}]$ & $T[$ days $]$ & $\theta_{E P}(0)\left[^{\circ}\right]$ & Feasiblity \\
\hline 3.196574 & 1.051307 & 4.247881 & 130.0962 & 58.831 & Yes \\
\hline 3.196574 & 1.051318 & 4.247892 & 44.9697 & 66.425 & Yes \\
\hline 3.196574 & 1.051319 & 4.247892 & 2.3911 & -135.535 & Yes \\
\hline 3.196574 & 1.051318 & 4.247892 & 98.3274 & 410.058 & Yes \\
\hline 3.196574 & 1.051307 & 4.247881 & 84.8219 & 203.858 & Yes \\
\hline 3.196574 & 1.051319 & 4.247893 & 72.3106 & 67.588 & Yes \\
\hline 3.196574 & 1.051307 & 4.247881 & 59.1727 & -145.870 & Yes \\
\hline
\end{tabular}

\section{Discussion}

Table 2 and Figures 1 and 2 show that trajectories with different times of flight can be found for the same Jacobi Integral value, which means that the fuel consumption, 
represented by $\Delta v_{\text {TOTAL }}$, is almost the same as shown in Table 2 . The procedure described by the TPBVP facilitates the determination of trajectories with large times of flight as seen in Figures 1 and 2 where trajectories with 202.17 and 284.02 days were obtained. Figures 3 and 4 compare an internal trajectory with an external trajectory with times of flight relatively the same. The external trajectory, Figure 4, has higher fuel consumption than the internal trajectory, Figure 3. This effect occurs due to the first velocity increment, which has to be at least $3.2 \mathrm{~km} / \mathrm{s}$ approximately for the space vehicle reach the Earth Sphere of Influence (SOI) in order to perform an external trajectory. Therefore, the internal trajectories generally consume less fuel than external trajectories considering the same time of flight. However, significant fuel consumption can be saved for external trajectories if the Sun influence is considered.

\section{Conclusions}

This work proposes a new method to determine Earth-Moon trajectories in the PCR3BP through a TPBVP with the Jacobi Integral previously specified. In this way, internal and external trajectories are obtained with different times of flight. The results show that this method facilitates the determination of trajectories with large times of flight. For future work, the trajectories can be optimized, and, the Sun influence can be analyzed.

\section{Acknowledgment}

The research was supported by Fapesp under contracts 2012/25308-5 and 2012/21023-6 and CNPq under contract 304913/2013-8.

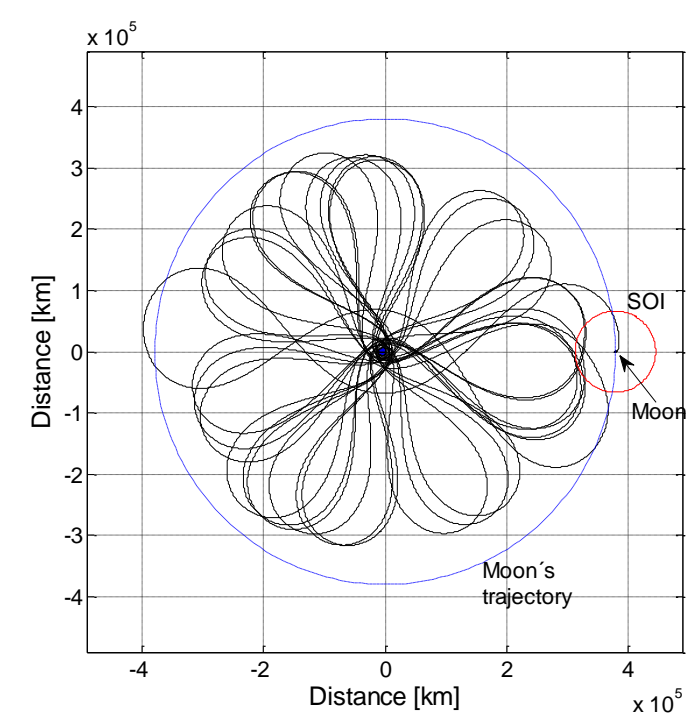

Figure 1: $C=2.90 \mathrm{~km}^{2} / \mathrm{s}^{2} . T=284.02$ days. $\Delta v_{\text {TOTAL }}=3.833027 \mathrm{~km} / \mathrm{s}$.

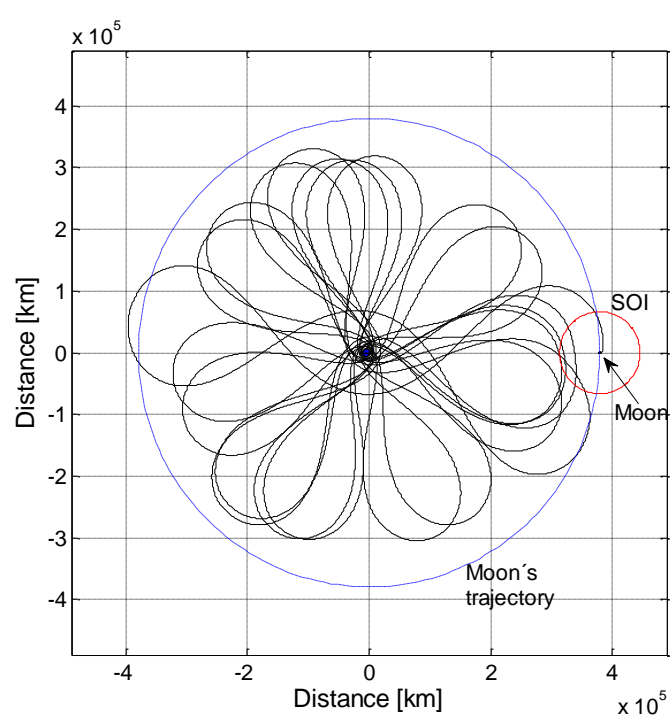

Figure 2: $C=2.90 \mathrm{~km}^{2} / \mathrm{s}^{2} . T=202.17$ days. $\Delta v_{T O T A L}=3.833027 \mathrm{~km} / \mathrm{s}$. 


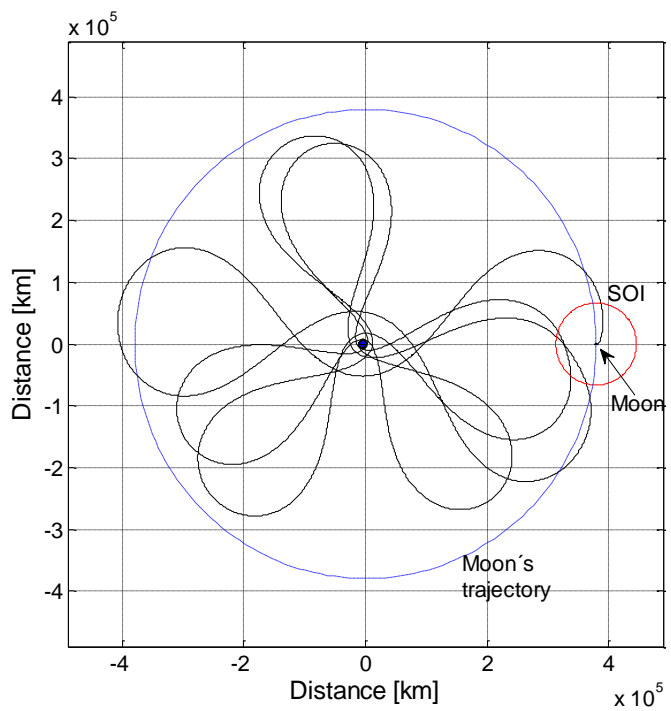

Figure 3: $C=2.80 \mathrm{~km}^{2} / \mathrm{s}^{2} . T=82.53$ days. $\Delta v_{\text {TOTAL }}=3.858772 \mathrm{~km} / \mathrm{s}$.

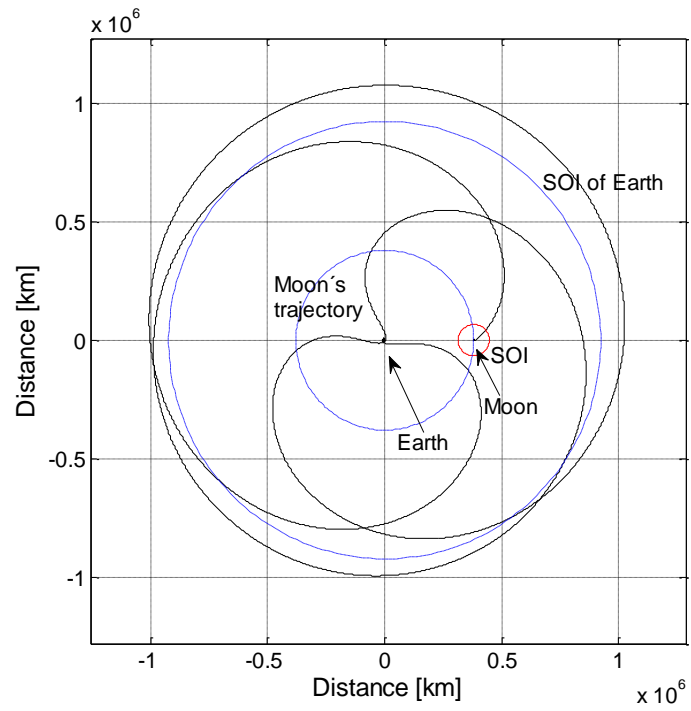

Figure 4: $C=1.20 \mathrm{~km}^{2} / \mathrm{s}^{2} . T=84.82$ days. $\Delta v_{\text {TOTAL }}=4.247880 \mathrm{~km} / \mathrm{s}$.

\section{Reference}

[1] E. A. Belbruno J. K. Miller, Sun-perturbed Earth-to-Moon transfers with ballistic capture, Journal of Guidance, Control, and Dynamics, vol. 16, n. 4, 770-775, (1993).

[2] S. Da Silva Fernandes S and C. M. P. Marinho, Optimal two-impulse trajectories with moderate flight time for earth-moon missions, Mathematical Problems in Engineering, (2012).

[3] S. Da Silva Fernandes and C. M. P. Marinho, Sun perturbations on optimal trajectories for Earth-Moon flight, Proceeding Series of the International Congress of Mechanical Engineering COBEM 2013. Ribeirão Preto, SP, Brazil: ABCM, (2013).

[4] Koon W S, et al., Low energy transfer to the Moon, Dynamics of Natural and Artificial Celestial Bodies, Springer, 63-73, (2001).

[5] J. P. Marec, Optimal space trajectories, New York: Elsevier, vol. 1, (1979).

[6] F. Topputo, On optimal two-impulse Earth-Moon transfers in a four-body model, Celestial Mechanics and Dynamical Astronomy, vol. 117, n. 3, 279-313, (2013).

[7] M. T. Zuber, et al, Gravity field of the Moon from the gravity recovery and interior laboratory (GRAIL) mission, Science, vol. 339, 668-671, (2013). 